\title{
Prognostic effects of osteoclast inhibitors in extensive stage small cell lung cancer patients with bone metastases
}

\author{
ATSUSHI MITSUHASHI ${ }^{1}$, YUSUKE OKUMA ${ }^{1,2}$, YOSHITAKA ZENKE ${ }^{1}$ and YUKIO HOSOMI ${ }^{1}$ \\ ${ }^{1}$ Department of Thoracic Oncology and Respiratory Medicine, Tokyo Metropolitan Cancer and \\ Infectious Diseases Center Komagome Hospital, Bunkyo, Tokyo 113-8677; ${ }^{2}$ Division of Oncology, \\ Research Center for Medical Sciences, Jikei University School of Medicine, Minato-ku, Tokyo 105-8461, Japan
}

Received April 24, 2018; Accepted August 31, 2018

DOI: $10.3892 /$ mco.2018.1710

\begin{abstract}
Bone metastases (BM) often induce skeletal-related events (SREs) and contribute to poor prognoses in patients with cancer. Osteoclast inhibitors (OIs), such as bisphosphonates (BPs) and denosumab, reportedly prevent SREs and improve quality of life in patients with non-small cell lung cancer and $\mathrm{BM}$, but have not been tested in extensive stage small cell lung cancer (ES-SCLC) patients. From 238 SCLC patient records, the present study reviewed those of $58 \mathrm{BM}$ patients, including 23 who were treated with OIs (OIs group) and 35 who were untreated (untreated group). Patient backgrounds were balanced between groups using propensity score matching, and survival curves were compared using the log-rank test. The median overall survival (OS) times were 8.41 and 12.52 months in untreated and OIs groups, respectively, but these did not differ significantly between groups (log-rank test, $\mathrm{P}=0.409$ ). The 1-year OS rate was higher in the OIs group (56.1\%) when compared with the control group (22.6\%). The results indicated that OIs tend to prolong the short term survival of ES-SCLC patients with BM. To the best of our knowledge, this is the first study to examine the prognostic effects of OIs in SCLC patients. The results of the present study may highlight the possibility that OIs improve the prognosis of ES-SCLC patients with BM.
\end{abstract}

\section{Introduction}

Skeletal tissues are among the most common sites of metastases from cancers such as breast, prostate, thyroid and lung carcinomas (1), and bone metastases (BM) reportedly occur in $15-30 \%$ of patients with lung cancer (2). BM strongly affect prognoses by causing skeletal-related events (SREs),

Correspondence to: Dr Yusuke Okuma, Department of Thoracic Oncology and Respiratory Medicine, Tokyo Metropolitan Cancer and Infectious Diseases Center Komagome Hospital, 3-18-22 Honkomagome, Bunkyo, Tokyo 113-8677, Japan

E-mail: y-okuma@cick.jp

Key words: bone metastases, osteoclast inhibitors, zoledronate, skeletal-related events, lung cancer such as pathological fracture, hypercalcaemia and spiral cord compression (3), and prevention of $\mathrm{BM}$ is a primary objective of treatments for non-small cell lung cancers (NSCLC).

Because osteoclasts induce bone resorption during the progression of BM (4), osteoclast inhibitors (OIs) are used as therapeutic agents, and bisphosphonates (BPs) and denosumab are commonly used to suppress SREs that are caused by BM. BPs are also commonly administered to inhibit bone resorption in solid tumour patients with BM (5). Among these agents, Zoledronate $(\mathrm{Zol})$ has been shown to delay or reduce the incidence of SREs in several solid tumours, including NSCLC (6). Furthermore, previous studies suggest that treatments with Zol improve overall survival (OS) and metastasis-free survival in NSCLC patients with BM (7), presumably be maintaining bone health. In addition, a recent study suggested that a monoclonal antibody against receptor activator of nuclear factor kappa-B ligand (RANKL), denosumab, is effective against NSCLC in patients with BM (8). Moreover, phase 3 trials of denosumab vs. Zol show significantly greater OS after treatments with denosumab than with Zol among patients with BM from lung cancers (9). These studies warrant the use of OIs for the prevention of SREs.

In contrast with treatments for NSCLC patients with BM, limited evidence is available to inform treatments for small cell lung cancer (SCLC) patients with BM. SCLC is an aggressive subtype of lung cancer and BM occurs in about $40 \%$ of extensive stage (ES)-SCLC patients. Moreover, BM contributes to poor prognoses among SCLC patients, as among NSCLC subjects, and OS of patients with BM is demonstrably poorer than that of patients without BM (10). However, no published studies report the prognostic efficacy of OIs in SCLC patient with BM.

Herein, we hypothesised that OIs prolong the survival of ES-SCLC patients by preventing the onset of SREs and reviewed the data from ES-SCLC patients with BM at Tokyo Metropolitan Cancer and Infectious diseases Center Komagome Hospital. Subsequently, we retrospectively evaluated the efficacy of OIs in ES-SCLC patients with BM.

\section{Materials and methods}

Data acquisition. Data were retrospectively collected from records of patients with ES-SCLC and BM who were admitted to Tokyo Metropolitan Cancer and Infectious diseases Center 
Komagome Hospital between 2008 and 2015. Among a total of 238 patients with SCLC, 132 patients were diagnosed with ES-SCLC and 58 of these had BM during the observation period and were enrolled in the study. Records of sex, age, smoking history, Eastern Cooperative Oncology Group scale performance status (ECOG PS) at the time of diagnosis of lung cancer, serum lactate dehydrogenase (LDH), date of treatment with OIs, date of onset of SREs, date of last follow up and patient outcomes were reviewed, and patients were assigned to treatment ( $\mathrm{Zol}$ and/or denosumab, $\mathrm{n}=23$ ) and non-treatment groups $(n=35)$. The decision of OIs treatment was based on physician's choice. The patients received pre-therapy dental care to prevent OIs related osteonecrosis of the jaw. OIs treatment was postponed until the completion of dental surgery. SREs were used as a primary outcome and included pathologic fracture, radiation to BM, surgery to bone, spinal cord compression and hypercalcaemia. All study protocols were approved by the Institutional Review Committee of Tokyo Metropolitan Cancer and Infectious diseases Center Komagome Hospital.

Statistical analysis. Descriptive statistics were used to summarise baseline characteristics. OS was defined as the time from the date of SCLC diagnosis to the date of death by any cause. Times to SREs from BM were not determined because almost all patients in the OIs group were treated after the onset of SREs. Categorical variables were compared using Pearson's, chi-square or Fisher's exact tests. Log-rank analyses were performed to compare OS using the Kaplan-Meier method, and background intergroup differences were minimised by calculating propensity scores based on patient characteristics. $\mathrm{P}<0.05$ was considered to indicate a statistically significant difference. All statistical analyses were performed using $\mathrm{R}$ version 3.2.2 (www.r-project.org/).

\section{Results}

Patients characteristics.Patient characteristics are summarised in Table I. The median age of patients was 70 years, and the median of Brinkman index was 1,080 . SREs were observed in 24 of 58 patients (41.4\%), and 35 of 58 patients with BM had never been treated been with OIs. The OIs group included 15 patients who were treated with ZOL and six who were treated with denosumab. The remaining two patients were administrated $\mathrm{Zol}$ and denosumab in combination. Palliative radiotherapy was the most common (83.3\%) SRE in patients with BM (Table II), and in comparisons of baseline characteristics between groups (Table III), SREs occurred more frequently in the control group than in patients treated with OIs, presumably because OIs were often administered after the incidence of SREs. In addition, recurrence and loss to follow up rates differed between control and OIs groups. To accommodate these differences, we performed propensity score matching (PSM) for age, sex, serum LDH, PS (ECOG), smoking history and recurrence, and subsequently found no significant differences in the incidence of SREs between untreated and OIs treated patients.

Survival analysis. The median OS of 58 patients with BM was 8.87 months, whereas that of 74 ES-SCLC patients
Table I. Baseline characteristics of small cell lung cancer patients with bone metastases.

\begin{tabular}{|c|c|c|}
\hline Characteristics & $\begin{array}{c}\text { No. of } \\
\text { patients (n) }\end{array}$ & $\%$ of total (n) \\
\hline No. of patients & 58 & \\
\hline Age, years (median) & 70 & \\
\hline \multicolumn{3}{|l|}{ Gender } \\
\hline Male & 47 & 81.0 \\
\hline Female & 11 & 18.9 \\
\hline \multicolumn{3}{|l|}{ Smoking status } \\
\hline Never-smoked & 1 & 1.7 \\
\hline Smoker & 48 & 82.8 \\
\hline No data & 9 & \\
\hline Brinkman index (median) & 1,080 & \\
\hline \multicolumn{3}{|l|}{ ECOG performance status } \\
\hline $0-1$ & 29 & 50.0 \\
\hline 2 & 9 & 15.5 \\
\hline $3-4$ & 17 & 29.3 \\
\hline No data & 3 & \\
\hline \multicolumn{3}{|l|}{ SREs } \\
\hline No & 30 & 51.7 \\
\hline Yes & 24 & 41.4 \\
\hline No data & 4 & \\
\hline Serum LDH (U/l, median) & 287 & \\
\hline \multicolumn{3}{|l|}{ Treatment of OIs } \\
\hline Never treated & 35 & 60.3 \\
\hline Zol & 15 & 25.9 \\
\hline Denosumab & 6 & 10.3 \\
\hline Zol and Denosumab & 2 & 3.4 \\
\hline
\end{tabular}

ECOG, Eastern Cooperative Oncology Group; SREs, skeletal-related events; OIs, osteoclast inhibitors; Zol, zoledronate.

Table II. Skeletal-related events in 24 of 58 extensive stage-small cell lung cancer patients with bone metastasis

\begin{tabular}{lcc}
\hline SRE & $\begin{array}{c}\text { No. of } \\
\text { patients (n) }\end{array}$ & \% of total (n) \\
\hline Radiation to bone & 20 & 83.3 \\
Spinal cord compression & 4 & 16.7 \\
Pathologic fracture & 3 & 12.5 \\
Hypercalcemia & 1 & 4.2 \\
Surgical stabilization & 1 & 4.2
\end{tabular}

SRE, skeletal-related event.

without BM was 11.04 months ( $\mathrm{P}=0.496$, Fig. 1). Kaplan-Meir analyses showed that $\mathrm{BM}$ is not a significant prognostic factor in comparison with metastases in other organs. In addition, no significant survival differences were identified between patients with $(n=23)$ and without $(n=35)$ OIs treatments 
Table III. Baseline characteristics of patients with and without osteoclast inhibitor treatments.

\begin{tabular}{|c|c|c|c|c|c|c|}
\hline \multirow[b]{2}{*}{ Characteristics } & \multicolumn{3}{|c|}{ Prior to PSM (n) } & \multicolumn{3}{|c|}{ Following PSM (n) } \\
\hline & With OIs & Without OIs & P-value & With OIs & Without OIs & P-value \\
\hline No. of patients & 23 & 35 & & 19 & 19 & \\
\hline Age, years (median) & 67 & 70 & 0.373 & 67 & 70 & 0.359 \\
\hline Gender & & & 0.316 & & & 1 \\
\hline Male & 17 & 30 & & 15 & 15 & \\
\hline Female & 6 & 5 & & 4 & 4 & \\
\hline Smoking status & & & 0.961 & & & 0.364 \\
\hline Never-smoked & 0 & 1 & & 0 & 1 & \\
\hline Smoker & 20 & 28 & & 19 & 18 & \\
\hline No data & 3 & 6 & & 0 & 0 & \\
\hline Brinkman index (median) & 1,080 & 1,080 & & 1,140 & 1,000 & \\
\hline ECOG performance status & & & 0.092 & & & 0.145 \\
\hline $0-1$ & 14 & 15 & & 12 & 8 & \\
\hline 2 & 3 & 6 & & 2 & 3 & \\
\hline $3-4$ & 5 & 12 & & 5 & 8 & \\
\hline No data & 1 & 2 & & 0 & 0 & \\
\hline SREs & & & 0.027 & & & 0.310 \\
\hline No & 8 & 22 & & 8 & 10 & \\
\hline Yes & 14 & 10 & & 11 & 6 & \\
\hline No data & 1 & 3 & & 0 & 3 & \\
\hline Serum LDH (U/l, median) & 297 & 271 & 0.511 & 288 & 256 & 0.839 \\
\hline
\end{tabular}

ECOG, Eastern Cooperative Oncology Group; SREs, skeletal-related events; OIs, osteoclast inhibitors; PSM, propensity score matching.

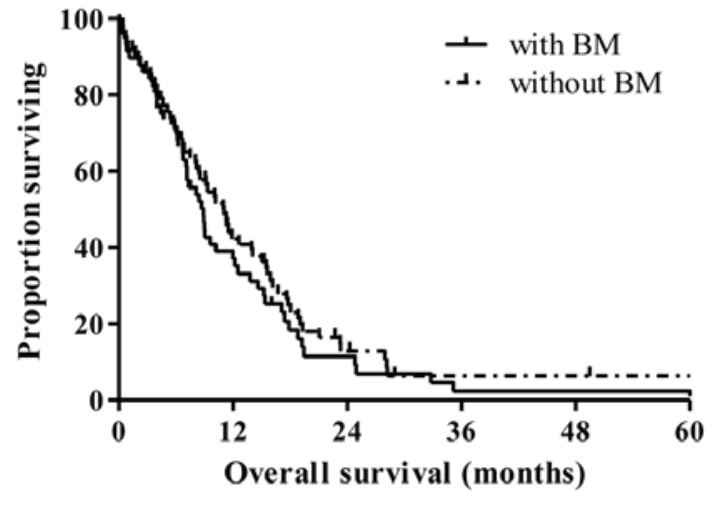

Patients at risk

\begin{tabular}{lcccccc}
\hline With BM & 58 & 20 & 6 & 2 & 2 & 2 \\
Without BM & 74 & 28 & 8 & 3 & 3 & 2
\end{tabular}

Figure 1. Survival of patients with stage IV small cell lung cancer with and without BM. BM, bone metastasis.

(median OS, 8.41 vs. 12.52 months, respectively; $\mathrm{P}=0.353$; Fig. 2A). However, one-year OS rate in OIs group were much higher compared to control group (55.1 vs. 24.7\%). These data suggest a tendency for prolonged survival of ES-SCLC patients treated with OIs, mainly in the early phase within the observation period of one year. Finally, matched-pair analyses using propensity scores confirmed the absence of a significant difference between the groups of the present study (median OS, 8.41 vs. 12.52 months, $\mathrm{P}=0.409$, Fig. 2B). One-year OS rate in OIs group were also higher compared to control group after PSM (56.1 vs. 22.6\%).

\section{Discussion}

BM is a well-known negative prognostic factor in SCLC and NSCLC patients, and a previous retrospective analysis of SCLC patients showed significantly shorter OS of patients with $\mathrm{BM}$ than among patients without BM [4.13 vs. 6.17 months, $\mathrm{P}=0.015$ (11)]. In NSCLC patient cohorts, the incidence of SREs is also a known negative prognostic factor, with one year survival rates of 5.1 and $12.1 \%$ in a retrospective cohort study of lung cancer patients with and without SREs, respectively (12). However, this study showed no significant differences in OS between ES-SCLC patients with BM and metastasis in the brain, liver, adrenal glands and pleural cavities. Other studies suggest that liver metastases are the worst prognostic factor in patients with ES-SCLC $(13,14)$, and that BM and brain metastases are similarly negative survival indicators. Specifically, one-year survival of SCLC patients with liver, brain and BM was $14.5,25.1$ and $23.9 \%$, respectively (13), warranting consideration of BM as an important therapeutic target in SCLC patients.

In the present study, OIs tended to prolong the survival of SCLC patients with BM in the short term, but our data failed 
A

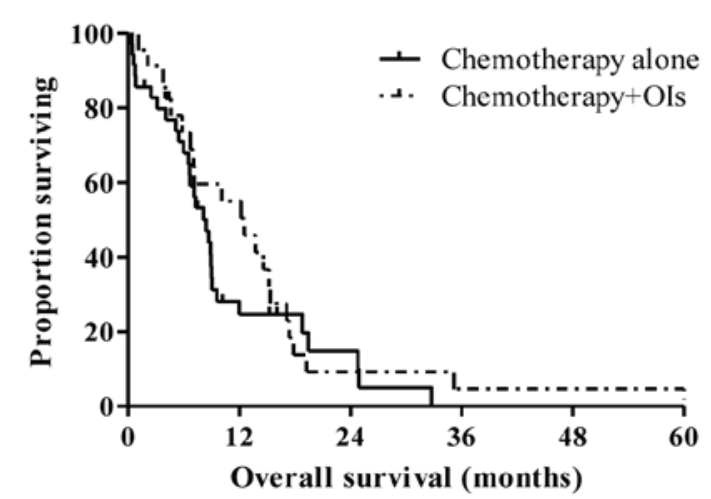

Patients at risk

\begin{tabular}{lrrrrrr}
\hline Chemotherapy alone & 35 & 8 & 4 & 1 & 1 & 1 \\
Chemotherapy +OIs & 23 & 13 & 3 & 2 & 2 & 2
\end{tabular}

B

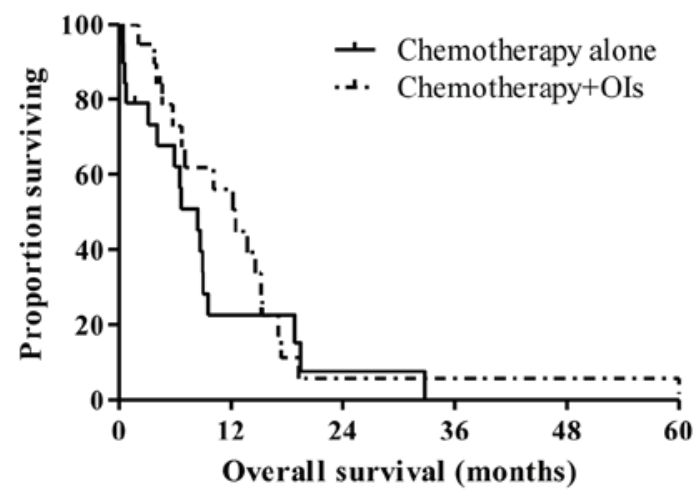

\begin{tabular}{rrrrrr}
\hline 19 & 5 & 2 & 1 & 1 & 1 \\
19 & 11 & 2 & 2 & 2 & 2
\end{tabular}

Figure 2. Comparison of survival between OIs treated and untreated small cell lung cancer patients with BM (A) prior to and (B) following PSM. BM, bone metastasis; OI, osteoclast inhibitor; PSM, propensity score matching.

to demonstrate a significant difference between the groups, likely reflecting insufficient numbers of cases in the OIs group, as OIs were not generally administered to SCLC patients as they were to NSCLC patients. In a recent prospective study, BM occurred in $39.5 \%$ of NSCLC patients who were treated with Zol, whereas only $6.7 \%$ of ES-SCLC patients developed $\mathrm{BM}$ in the same period (10), potentially reflecting the high chemotherapeutic sensitivity of SCLC. In other studies, overall response rates to combination therapy with VP-16 or etoposide and cisplatin (EP) were 86-89\% in ES-SCLC patients $(15,16)$.

In the present study, most patients in the OIs group were treated in response to SREs. Hence, appropriate administration of OIs before the onset of SREs may decrease the frequency of SREs and improve OS in SCLC patients with BM. However, future large-scale prospective studies are required to precisely define the benefits of OIs in SCLC patients with BM.

Although the incidence of SREs was not decreased by OIs in the present SCLC patients with BM, likely reflecting retrospective treatments, new incidences of SREs were observed in 10 of the 23 OIs treated patients, suggesting that OIs prolong survival via a mechanism that is independent of the suppression of SREs. In agreement, Zol reportedly suppressed the proliferation of SCLC cell lines in vitro and in vivo $(17,18)$, and these antitumor effects of Zol were associated with inhibition of farnesylation and geranylgeranylation of RAS related proteins, and the induction of apoptosis (17). Zol also showed synergistic effects with other anti-cancer agents, such as cisplatin, etoposide and irinotecan in vitro (19-21), and a recent preclinical study showed that Zol promotes the maturation of $\gamma \delta$ T cells and contributes to antitumor immune responses (22). Thus, Zol may improve the prognoses in SCLC patients via direct cytotoxic mechanisms or antitumor immunity, in addition to acting as an inhibitor of SREs.

Denosumab is also expected to have direct antitumor effects, because Its target RANK/RANKL signalling is known to activate NF- $\kappa \mathrm{B}$, which has been associated with carcinogenesis, resistance to chemoradiotherapy and metastasis (8).
Specifically, RANKL is expressed on several tumour types, including lung cancers (23), and therefore, RANK/RANKL signalling has potential as a therapeutic target. However, RANKL expression rates have not yet been determined in SCLC, warranting further studies of direct and indirect antitumor effects of denosumab in this patient group.

Our analyses were limited to retrospective data from a single institution with relatively few cases, especially in the OIs group. In addition, we failed to distinguish between the effects of Zol and denosumab as independent groups, again reflecting the small number of cases treated with denosumab and those cotreated with Zol. Finally, durations of OIs treatments varied among the present cases, and the start times were not determined with reference to occurrences of SREs. Hence, prospective studies are required to confirm the relationships between preventative effects against SREs and survival.

The present data suggest that OIs prolong the survival period of ES-SCLC patients with BM in the short term and warrant larger prospective studies to inform the use of OIs as preventative treatments for SREs in ES-SCLC patients with BMs.

\section{Acknowledgements}

The authors would like to thank Dr Makoto Saito (Division of Clinical Research Support, Tokyo Metropolitan Cancer and the Infectious Diseases Center Komagome Hospital, Tokyo, Japan), for his statistical expertise.

\section{Funding}

No funding was received.

\section{Availability of data and materials}

All data generated or analyzed during this study are included in this published article. 


\section{Authors' contributions}

AM and YO analyzed the data of the SCLC patients with bone metastasis, and were major contributors in writing this manuscript. $\mathrm{YZ}$ and $\mathrm{YH}$ analyzed and interpreted the patient data. All authors read and approved the final manuscript.

\section{Ethics approval and consent to participate}

All study protocols were approved by the Institutional Review Committee of Tokyo Metropolitan Cancer and Infectious diseases Center Komagome Hospital (Tokyo, Japan); the requirement for written informed consent was waived due to the retrospective nature of this study.

\section{Patient consent for publication}

Not applicable.

\section{Competing interests}

The authors declare that they have no competing interests.

\section{References}

1. Mundy GR: Metastasis to bone: Causes, consequences and therapeutic opportunities. Nat Rev Cancer 2: 584-593, 2002.

2. Coleman RE: Clinical features of metastatic bone disease and risk of skeletal morbidity. Clin Cancer Res 12: 6243s-6249s, 2006.

3. Brodowicz T, O'Byrne $\mathrm{K}$ and Manegold C: Bone matters in lung cancer. Ann Oncol 23: 2215-2222, 2012.

4. Roodman GD: Mechanisms of bone metastasis. N Engl J Med 350: 1655-1664, 2004

5. Serafini AN: Therapy of metastatic bone pain. J Nucl Med 42: 895-906, 2001

6. Rosen LS, Gordon D, Tchekmedyian NS, Yanagihara R, Hirsh V, Krzakowski M, Pawlicki M, De Souza P, Zheng M, Urbanowitz G, et al: Long-term efficacy and safety of zoledronic acid in the treatment of skeletal metastases in patients with nonsmall cell lung carcinoma and other solid tumors: A randomized, Phase III, double-blind, placebo-controlled trial. Cancer 100: 2613-2621, 2004.

7. Zarogoulidis K, Boutsikou E, Zarogoulidis P, Eleftheriadou E, Kontakiotis T, Lithoxopoulou H, Tzanakakis G, Kanakis I and Karamanos NK: The impact of zoledronic acid therapy in survival of lung cancer patients with bone metastasis. Int $\mathbf{J}$ Cancer 125: 1705-1709, 2009.

8. De Castro J, García R, Garrido P, Isla D, Massuti B, Blanca B and Vázquez J: Therapeutic potential of denosumab in patients with lung cancer: Beyond prevention of skeletal complications. Clin Lung Cancer 16: 431-446, 2015.

9. Scagliotti GV, Hirsh V, Siena S, Henry DH, Woll PJ, Manegold C, Solal-Celigny P, Rodriguez G, Krzakowski M, Mehta ND, et al: Overall survival improvement in patients with lung cancer and bone metastases treated with denosumab versus zoledronic acid: Subgroup analysis from a randomized phase 3 study. J Thorac Oncol 7: 1823-1829, 2012.
10. Katakami N, Kunikane H, Takeda K, Takayama K, Sawa T, Saito H, Harada M, Yokota S, Ando K, Saito Y, et al: Prospective study on the incidence of bone metastasis (BM) and skeletal-related events (SREs) in patients (pts) with stage IIIB and IV lung cancer-CSP-HOR 13. J Thorac Oncol 9: 231-238, 2014.

11. Kang EJ, Lee SY, Kim HJ, Min KH, Hur GY, Shim JJ, Kang KH, Oh SC, Seo JH, Lee SY and Kim JS: Prognostic factors and skeletal-related events in patients with small cell lung cancer with bone metastases at the time of diagnosis. Oncology 90: 103-111, 2016.

12. Cetin K, Christiansen CF, Jacobsen JB, Nørgaard $M$ and Sørensen HT: Bone metastasis, skeletal-related events, and mortality in lung cancer patients: A Danish population-based cohort study. Lung Cancer 86: 247-254, 2014.

13. Ren Y, Dai C, Zheng H, Zhou F, She Y, Jiang G, Fei K, Yang P, Xie D and Chen C: Prognostic effect of liver metastasis in lung cancer patients with distant metastasis. Oncotarget 7: 53245-53253, 2016

14. Ogino H, Hanibuchi M, Kakiuchi S, Saijo A, Tezuka T, Toyoda Y, Tobiume M, Otsuka K, Sakaguchi S, Goto H, et al: Analysis of the prognostic factors of extensive disease small-cell lung cancer patients in Tokushima University Hospital. J Med Invest 63: 286-293, 2016.

15. Goldhirsch A, Joss R, Cavalli F and Brunner KW: Etoposide as single agent and in combination chemotherapy of bronchogenic carcinoma. Cancer Treat Rev 9: 85-90, 1982.

16. Evans WK, Osoba D, Feld R, Shepherd FA, Bazos MJ and DeBoer G: Etoposide (VP-16) and cisplatin: An effective treatment for relapse in small-cell lung cancer. J Clin Oncol 3: 65-71, 1985.

17. Matsumoto S, Kimura S, Segawa H, Kuroda J, Yuasa T, Sato K, Nogawa M, Tanaka F, Maekawa T and Wada H: Efficacy of the third-generation bisphosphonate, zoledronic acid alone and combined with anti-cancer agents against small cell lung cancer cell lines. Lung Cancer 47: 31-39, 2005.

18. Kenessey I, Kói K, Horváth O, Cserepes M, Molnár D, Izsák V, Dobos J, Hegedús B, Tóvári J and Tímár J: KRAS-mutation status dependent effect of zoledronic acid in human non-small cell cancer preclinical models. Oncotarget 7: 79503-79514, 2016.

19. Neville-Webbe HL, Evans CA, Coleman RE and Holen I: Mechanisms of the synergistic interaction between the bisphosphonate zoledronic acid and the chemotherapy agent paclitaxel in breast cancer cells in vitro. Tumour Biol 27: 92-103, 2006.

20. Ozturk OH, Bozcuk H, Burgucu D, Ekinci D, Ozdogan M, Akca S and Yildiz M: Cisplatin cytotoxicity is enhanced with zoledronic acid in A549 lung cancer cell line: Preliminary results of an in vitro study. Cell Biol Int 31: 1069-1071, 2007.

21. Budman DR and Calabro A: Zoledronic acid (Zometa) enhances the cytotoxic effect of gemcitabine and fluvastatin: In vitro isobologram studies with conventional and nonconventional cytotoxic agents. Oncology 70: 147-153, 2006.

22. Fournier PG, Chirgwin JM and Guise TA: New insights into the role of T cells in the vicious cycle of bone metastases. Curr Opin Rheumatol 18: 396-404, 2006.

23. Peng X, Guo W, Ren T, Lou Z, Lu X, Zhang S, Lu Q and Sun Y: Differential expression of the RANKL/RANK/OPG system is associated with bone metastasis in human non-small cell lung cancer. PLoS One 8: e58361, 2013. 